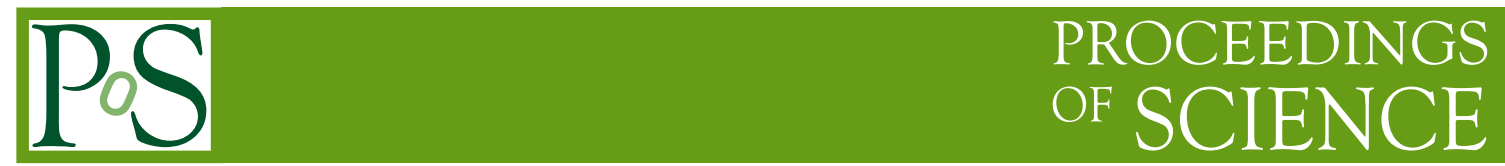

\title{
Status of direct search of WIMP Dark Matter
}

\section{Marcello MESSINA*}

Columbia University, New York, NY, USA

E-mail: mmessina@astro.columbia.edu

In this paper we give an overview of the experiments in the field of direct Dark Matter search. The physics topic is introduced starting with some historical information and then the experiments, which exploit the most commonly used technologies, are quickly described together with the main features and the expected sensitivity. Not all the experiments are mentioned but attention is put on those that the author judges they give the most relevant contributions in the field.

Neutrino Oscillation Workshop

4 - 11 September, 2016

Otranto (Lecce, Italy)

\footnotetext{
*Speaker.
} 


\section{Introduction}

In the framework of the Cosmological Standard Model (CSM) it is well assessed the presence in the Universe of a sizable fraction of matter composed by particles not belonging to the Standard Model (SM) of Particle Physics. The discussion on the possible presence of an unexpected matter component of the Universe started in 1922 with Jacobus Cornelius Kapteyn [1] which was the first to realize that the Milky Way (MW) rotates as contrary to the common believe, of that time, according to which the stars were moving randomly. Subsequently, in 1932 Jan Oort determined the MW center of rotation overpassing definitively the idea that the Sun was the center of the MW. Finally in 1933 Fritz Zwicky realized, by measuring the velocity of galaxies in the Coma clusters, that those were larger than the escape velocity accounted by visible matter [2]. Thus, he came to the hypothesis of the presence of "Dunkle Materie", that today is indicated as Dark Matter (DM), which would provide the gravitation potential to keep together the visible matter. DM was only known to be some form of matter, non emitting light and interacting gravitationally. Later on, a similar measurement was realized by Vera Rubin [3] on the M31 galaxy, showing that, the rotational velocity of stars was higher than what she was able to predict given the visible matter. Also at galaxy scale the presence of DM is needed to explain the velocity of stars.

The measurements of temperature anisotropy of the Cosmological Microwave Background (CMB) have been of crucial importance in the process of narrowing down the features of DM. The most recents measurements have been provided by WMAP and PLANCK experiments in [4] and [5], respectively. The CMB anisotropy imposes that a component of matter, not interacting with ordinary matter by means of electromagnetic forces, exists and it must have been present also in the early stages of the Universe. If DM is assumed to be stable it turns out to contribute to the Universe energy balance by $25 \%$ ( $85 \%$ if normalized to the matter content). The CMB measurements allowed to definitively establish that DM is not a form of non-luminous ordinary matter but some form of particles weakly interacting with those of the SM.

\section{Detection principle of DM}

A seminal paper where the DM detection was envisaged is [6]. The authors considered several possible candidates and among those also the neutrino's super-partner in a Supersymmetric extension of the SM, that is today the most commonly assumed DM constituent. In this framework DM particles are indicated as Weakly Interacting Massive Particle (WIMP). For a detailed treatment of this topic see [7]. In [6] the authors discuss the coherent scattering of DM particles and the possibility to detect very small energy release due to the scattering of WIMPs, with mass in the range of $1-10^{2} \mathrm{GeV}$. The cross sections of DM particles with ordinary matter is evaluated in both cases, spin dependent and spin independent. In [6] energy release of few $\mathrm{keV}$ up to tens of $\mathrm{keV}$ is considered and this is what today is named as DM direct detection. It differs from the indirect detection, where secondary products of the WIMP and anti-WIMP annihilation are detected in outer-space detectors, focused on the detection of $\left.\gamma, \mathrm{p} / \bar{p}, e^{-} / e^{+}\right)$, or in ground based ones more focused on $v$ and anti- $v$ detection.

The WIMP interaction rates with ordinary matter have been calculated in different theoretical frameworks, [7], all ending up with very low interaction rate and none of them confirmed by any 
measurement, yet. It is commonly accepted that the scale of the cross sections of WIMPs is the one of weak interactions. To support this assumption one can look at [8] where it is deduced the formula connecting the WIMP density, the annihilation cross-section of the WIMP and anti-WIMP at the freeze-out (when the WIMP annihilation rate became smaller than the Universe expansion rate, then the WIMPs decoupled form the primordial plasma) and the contribution of the DM to the critical density of the Universe.

The low interaction cross section is also confirmed by the most recent experiments on direct DM search where the negative results imposes that the DM rate must be lower than 1 event $/ \mathrm{kg} / \mathrm{yr}$.

The low interaction rate and the very little energy released impose experimental challenges. In fact, suitable detection techniques must have an energy threshold as low as few keV.

The low expected rate imposes also to install the apparatus in a clean and "silent" environment where the flow of cosmic rays is significantly reduced like in a underground laboratory.

A relevant aspect to consider in the design phase of a detector is the capability to measure the radioactive traces in each components of the detector, in order to sort out those with the lowest level of contaminants which might generate fake events originated by gammas or neutrons. Given the aimed at sensitivity the level of radioactive contaminants required nowadays is less than $\mu \mathrm{Bq} / \mathrm{kg}$.

Another important signature of DM interaction is the time variation of the interaction rate which must show a seasonal modulation because of the Earth motion and so for the experiment in the galaxy. The Earth speed is almost $10 \%$ of the one of the solar system in the galaxy. Thus, the composition of motions generate a variation of the relative velocity between experiment and galaxy and so of the rate of DM in a possible experiment.

\section{Overview of the experiments for direct DM detection}

In many target suitable for DM experiments a WIMP scattering provokes the production of phonons (heat), photons and ionization electrons. An optimal experiment should measure the three forms of energy but unfortunately this is never the case. The field of DM search is populated by experiments able to measure one or two forms of energy. For a complete review DM experiments one can look at [9] and the references reported therein. When it will be required we will cite the original paper.

The reason why we linger on the topic of the forms of released energy is due to the fact that while phonons are proportional to the energy, the response of the detector to photons or ionization electrons strongly depends on $d E / d x$. This gives the possibility to distinguish between events originated by gammas or electrons and by those originated by alpha or nuclear recoils. Two measurable quantities are sufficient to provide the capability to distinguish among events with different $d E / d x$.

The DAMA experiment, held at the Laboratori Nazionali del Gran Sasso (LNGS, INFN laboratory in l'Aquila, Italy), exploits the technology of $\mathrm{NaI}$ ultra-pure crystals, at room temperature, read out by high efficiency photodetectors (PMT) on one side. The PMTs measure scintillation photons generated by energy released in NaI crystals. DAMA has reached nowadays 1.33 tonnes $\times$ year of total exposure (DAMA/NaI+DAMA/Libra) with $233 \mathrm{~kg}$ of crystals. This experiment plays a special role in the panorama of direct DM search. Indeed, the DAMA collaboration claims that DM interactions have been measured in the energy interval of $2-6 \mathrm{keV}$. Furthermore, the event rate shows a sinusoidal modulation with an amplitude of $\sim 1 \%$ above background, one year period 
and the maximum on June 2nd. All mentioned features are compatible with a signal generated by $\mathrm{DM}$. The fact that the variation is not observed above $6 \mathrm{keV}$, and it is only detected in the data sample of single scattering reduces significantly the probability that it might be due to environmental neutrons.

Unfortunately, this signal has never been confirmed by any experiment. In fact, XENON100 and CDMS (these detectors will be presented later) were the first experiments to exclude the DAMA signal, as due to nuclear recoil, by several order of magnitude on the cross section of WIMP interaction with nuclear matter with WIMP masses above few $5 \mathrm{GeV}$. For long time the tension between the two results was accommodated either by assuming a negligible cross section of WIMPs on XENON100 and CDMS target nuclear matters, Xe and Ge, respectively, or assuming the signal as due to WIMP interactions with the atomic electrons. This was due to the fact that the DAMA detector does not provide particle identification whereas XENON100 detector is capable to distinguish between nuclear or electron recoils at better than 1\%. Recently, the XENON100 has published the most sensitive analysis in which the hypothesis of WIMPs interacting with electrons is excluded ([10]) and so the yearly modulation of WIMPs interactions on electrons ([11]). Thus the DAMA signal remains unconfirmed.

A full campaigns of new experiments based on a DAMA-like technology has started, exploiting new generation of ultra-pure NaI crystals. At LNGS the SABRE experimental project is in the phase of proof of principle and it is based on high quantum efficiency PMTs reading out NaI crystals on both side to increase the light collection efficiency. Furthermore, veto detector, surrounding the vessel hosting the crystals will improve the background rejection capability. Experiment based on a similar approach are also ongoing at CANFRANC laboratory in Spain, ANAIS, and at the Yang Yang laboratory in South Corea, where the COSINE-100 experiments merges the two experimental efforts of DM-Ice and KIMs collaborations that have shown, in the phase of detector studies, good performances of apparatus with $\mathrm{NaI}$ and $\mathrm{CsI}$ crystals, respectively.

It is worth pointing out that to improve WIMP search for large masses $\left(M_{\text {WIMP }}>10 \mathrm{GeV}\right)$ large mass detectors are required whereas to improve the sensitivity for low WIMP masses (i.e. $M_{\text {WIMP }}<10 \mathrm{GeV}$ ) the energy threshold must be reduced.

A different category of detectors, so called cryogenic calorimeters, lead the field of DM search in the sensitivity region below $10 \mathrm{GeV}$ of WIMPs masses. This type of detectors measures phonons and the relation between released energy and the temperature variation $(\Delta T)$ is given by $\Delta T=$ $\frac{\Delta E}{C} e^{\frac{-t}{\tau}}$ where $C$ is the heat capacitance of the absorber (bulk of the calorimeter) and $\Delta E$ is the released energy. The time evolution of temperature is described by the exponential where in the formula of the time constant $\tau=C / G G$ is the heat conductance between the absorber and the thermal bath, whose typical temperature is $\sim 10 \mathrm{mK}$. The SuperCDMS detector is the last of a family called CDMS(I-II) installed in the Soudan mine in Minnesota, USA, that exploited Si and Ge crystals. SuperCDMS will be installed at the SNOWLAB in Canada. It exploits the technology of cryogenic calorimeter made of Ge crystals of $600 \mathrm{~g}$ equipped with devices capable to measure phonons and electrons.

A peculiar interleaving pattern of the sensors gives particle identification and the capability to select events taking place on the edge of the crystals with respect to those happening in the core of the crystals. With these type of detector it is hard to obtain target masses of tonnes thus the main feature is low energy threshold that gives good sensitivity at low WIMPs masses. 
The EDELWEISS experiment (at the Laboratoire Souterrain de Modane in France) exploit the same technology like the one of SuperCMDS, with $800 \mathrm{~g} \mathrm{Ge}$ crystals cooled at $18 \mathrm{mK}$, read out by temperature sensors based on a different technology with respect to those of SuperCDMS. The ionization electrons are measured as well and so particle identification is possible. Both SuperCDMS and EDELWEISS aim at achieving a threshold on released energy lower than $100 \mathrm{eV}$.

The last experiment we want to mention, in this field, is CRESST, held at LNGS in Italy. It exploits scintillation crystals like $\mathrm{CaWO}_{4}$, cooled down at $8 \mathrm{mK}$. The comparison between phonons measurements, by means of sensors similar to those of SuperCDMS, and the scintillation light measured by low temperature light sensors, provides particle identification and also space resolution. The crystals of $250 \mathrm{~g}$ are presently used and the energy threshold is $300 \mathrm{eV}$. A factor ten reduction in mass of crystals is envisaged to reduce the threshold by a factor three. The first prototypes have overcame the design goal showing $50 \mathrm{eV}$ threshold. In future CRESST experiment aims at achieving the best sensitivity in low WIMP mass region. So far no evidence of DM observation came form these experiments.

As already mentioned at large masses of WIMPs the best sensitivity is given by large mass detectors. In this field the challenge of DM direct search is lead by detectors using liquefied Noble gases such as liquid $\mathrm{Ar}$ (LAr) and liquid Xe (LXe). These apparatuses are divided in two main categories single and double phase detectors, respectively.

DEAP-3600 is a single phase LAr detector exploiting $3600 \mathrm{~kg}$ of LAr in a spherical cryostat surrounded by an instrumented water detector (veto detector). The scintillation light of LAr, generated by particle interactions, is detected by means of 255 high quantum efficiency PMTs once the the Ar scintillation light (at $128 \mathrm{~nm}$ ) has been shifted in the visible region to match the efficiency curve of PMTs. The scintillation of Ar comes from singlets and triplets molecular states which decay with very much different decay times. The amount of light decaying with fast (ns) and slow decay time $(\mu \mathrm{s})$ depends strongly on $d E / d x$ allowing a high efficiency particle identifications. The use of Ar has the drawback that it comes with an isotope, ${ }^{39} \mathrm{Ar}$, which gives roughly one hertz of beta decay per $\mathrm{kg}$ of natural Ar. This reduces significantly the DM discovery potential of the Ar based detectors. In fact, all the apparatuses exploiting Ar target plan to use the so called depleted Ar, where the unstable isotope has been removed by means of distillation processes or by underground gas-production plant, where Ar comes without its unstable isotope.

On the contrary, X-MASS detector exploits $830 \mathrm{~kg}$ of LXe, in a spherical copper cryostat, instrumented by 642 PMTs for a $62 \%$ photocathode coverage. The large photocathode coverage provides position sensitivity allowing to select those events taking place in the center of the active volume and reject those happening on the edge. This is a crucial feature to exploit the fact that the Xe is very clean, has no long-lived unstable isotope, and has a perfect self-screening capability. Unfortunately, the fast and slow decay time features in the Xe provides very poor distinction capability because of very similar time constants.

An evolution of such a type of detector concept is the double phase liquefied noble gas detectors using either Ar or Xe. Both LAr and LXe, when energy is released in the active volume, create scintillation and ionization. A drift E-fields prevent the full electrons recombination with the parent nuclei and drift the electrons to the liquid-gas interface. A higher amplitude E-field extracts the electrons to the gaseous phase, accelerate them and generate a second light pulse. The two light pulses are detected by PMTs and the time difference between the two pulses allow to measure the 
depth or the coordinate across the drift field. The most suited geometry is a cylindrical one where the active volume is closed by a cathode and an anode. Field shapers are used to make the drift field as uniform as possible. The light pattern of the second pulse allow to reconstruct the transverse coordinates, orthogonal to the drift field direction. Thus a complete space reconstruction of events is possible. This detector takes the name of double phase Time Projection Chamber (TPC).

The XENON collaboration lead the field of LXe double phase TPC, building and running several detectors, at the LNGS, of increasing sensitivity, XENON10 and XENON100. The last one, still in the phase of analyzing the data, has been the detector with the best sensitivity until 2013, while the third one, XENON1T, is expected to lead the field for the next years. The XENON1T detector, with 3.5 tonnes of instrumented mass and more than 1 tonnes of fiducial mass, is presently starting the physics data taking. The expected sensitivity is such that the minimum cross section $2 \times 10^{-47} \mathrm{~cm}^{2}$ for a WIMP mass of $50 \mathrm{GeV}$ will be reached in two years of DM data-taking. The XENON1T has embedded in its design the possibility to upgrade the instrumented mass to 7 tonnes (XENONnT), reutilizing all the ancillaries devices of XENON1T, replacing only the vessel containing LXe, building a larger TPC with 100 more PMTs with respect to XENON1T.

The LUX experiment, usesig the same XENON technology, is located at the Sanford Laboratory in South Dakota, USA, is the direct competitor of XENON100 with $250 \mathrm{~kg}$ of fiducial mass that in 2013 has published the best results. In the most recent update ([12]) it has been shown an exclusion limit (at $90 \%$ C.L.) with a minimum cross section of $0.6 \times 10^{-45} \mathrm{~cm}^{2}$ at $33 \mathrm{GeV}$ WIMP masses after $1.41 \times 10^{4} \mathrm{~kg} \mathrm{x}$ day exposure. Also the LUX collaboration is designing an upgrade of the detector called LZ with an instrumented mass of around 7-10 tonnes. LZ is still in a design phase.

The PandaX-II, built and run a LXeTPC at the Jin-Ping laboratory in China, with $500 \mathrm{~kg}$ instrumented mass has published ([13]) an exclusion limit (at 90\% C.L.) with a minimum of $0.25 \times$ $10^{-45} \mathrm{~cm}^{2}$ at $40 \mathrm{GeV}$ WIMP mass after 98.7 days of data-taking.

Before to conclude with Xe based experiments it is worth noticing that thanks to the unpaired nucleons in the Xe nuclei (this is not true for the Ar) with these detectors is possible to achieve a sensitivity to spin dependent WIMP cross section.

Experiments using double phase LArTPC are also very active in the field of direct DM search. The leading one is the DarkSide-50 experiment, running at LNGS which has proved the principle and has shown the very high particle identification capability that can be achieved by exploiting the short and slow decay time constant. Recently the first results obtained with depleted Ar have been published. The same collaboration proposed a multi-ton detector ( $>20$ tonnes) aiming at achieving the best sensitivity in the field of direct DM search in the next 20 years. The capability to reach the desired goal depends largely on the fact that the collaboration will prove to have the technology for producing the required amount of depleted Ar, i.e. tonnes of Ar with a significant reduction of ${ }^{39} \mathrm{Ar}$. A program is under development to establish the technology of distillation of ${ }^{39} \mathrm{Ar}$ from natural $\mathrm{Ar}$ from air on tonnes scale.

To conclude, the plots in Fig. 1 summarize the situation of the most important exclusion curves from experiments that did not find any positive results and excluded the DAMA observation, which is presently the only one claiming DM signal observation. 

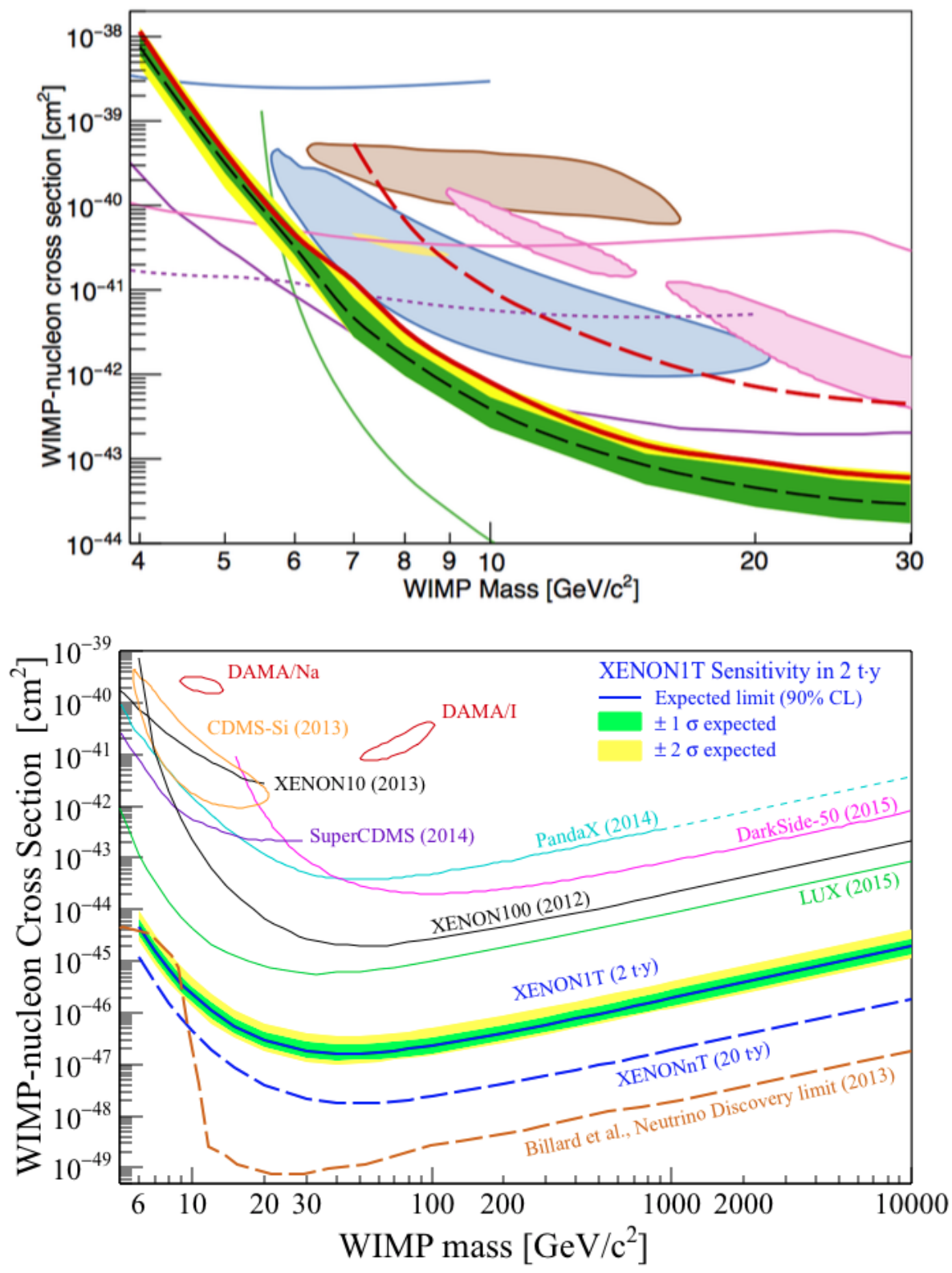

Figure 1: The plots above show the most updated results either in terms of discovery claim or exclusion limit in the region of sensitivity. For low WIMP masses $(<30 \mathrm{GeV})[14]$, top figure, the legenda is: red curve, green and yellow bands represent the exclusion limit at 90\% C.L. and the sensitivity regions at 1 and $2 \sigma$ in absence of signal of EDELWEISS-III, whereas, yellow, blue, pink and brown contours are the claims of discovery by CoGeNT, CDMS-Si, CRESST-II and DAMA, respectively. The limits by EDELWEISS-II (dashed red), LUX (green), DAMIC (blue), CRESST (pink), CDMSLite (dashed violet) and SuperCDMS are also shown. For large WIMP masses ( $>6 \mathrm{GeV}$ to $10 \mathrm{TeV})[15]$, bottom figure, the plot is self-explaining. In both cases the claims of Dark Matter observations are excluded. The aimed at exclusion limit of the experiments with the best projected sensitivity are also shown.

\section{Conclusion}

The claim of DM observation from the DAMA collaboration requires desperately a confirma- 
tion or a clear disclaim. The DM is the physics topic where the largest number of experiments are involved in the challenge in any corner of the space of parameters. We think that there are good chances to come to a result in the next 10 years.

\section{References}

[1] J. C. Kapteyn, Astrophys. J. 55, 302 (1922).

[2] F. Zwicky, Helv. Phys. Acta 6, 110 (1933).

[3] V. C. Rubin and W. K. Ford Jr, Astrophys. J. 159, 379 (1970).

[4] G. Hinshaw et al. (WMAP Collaboration), Astrophys. J. Suppl. 208, 1 (2013).

[5] R. Adam et al. (PLANCK Collaboration), Astro. and Astrophys., 594, 1 (2016).

[6] M. W. Goodman and E. Witten, Phys. Rev. D 31, 3059 (1985).

[7] G. Jungman, M. Kamionkowski and K. Griest, Phys. Rept. 267, 195 (1996).

[8] S. Dodelson, Modern Cosmology, Academic Press (2003).

[9] T. Marrodán Undagoitia and L. Rauch, J. Phys. G 43, 013001 (2016).

[10] E. Aprile et al. (XENON collaboration), Science 349, 851 (2015).

[11] E. Aprile et al. (XENON Collaboration), Phys. Rev. Lett. 115, 091302 (2015).

[12] D. S. Akerib et al. (LUX Collaboration), Phys. Rev. Lett. 116, 161102 (2016).

[13] A. Tan et al. (Panda-X II Collaboration), Phys. Rev. Lett. 117, 121303 (2016).

[14] E. Armengaud et al.(EDELWEISS-III Collaboration), JCAP 05, 19 (2016).

[15] E. Aprile et al. (XENON Collaboration), JCAP 04, 27 (2016). 